Research Article

\title{
Combination of Xuesaitong and Aspirin Based on the Antiplatelet Effect and Gastrointestinal Injury: Study Protocol for a Randomized Controlled Noninferiority Trial
}

\author{
Bao-Chen Zhu, ${ }_{1}^{1}$ Chun-Miao Xue, ${ }^{1}$ Rui Lang, ${ }^{2}$ Wei-Liang Weng, ${ }^{3,4}$ Xu-Jie Wang, ${ }^{3,4}$ \\ Zhen-Zhen Lei, ${ }^{1}$ Sha-Sha Zhang, ${ }^{1}$ Wen-Hua Yang, ${ }^{1}$ Wan-Tong Zhang $\mathbb{B},{ }^{3,4}$ \\ and Guo-Dong Hua $\mathbb{D}^{1}$ \\ ${ }^{1}$ Department of Pharmacy, Dongzhimen Hospital, Beijing University of Chinese Medicine, Beijing, China \\ ${ }^{2}$ Department of Nephrology, Xiyuan Hospital, China Academy of Chinese Medical Sciences, Beijing, China \\ ${ }^{3}$ Institute of Clinical Pharmacology, Xiyuan Hospital, China Academy of Chinese Medical Sciences, Beijing, China \\ ${ }^{4}$ National Clinical Research Center for Chinese Medicine Cardiology, Beijing, China
}

Correspondence should be addressed to Wan-Tong Zhang; wantong_zhang@hotmail.com and

Guo-Dong Hua; zhaojhuagd@126.com

Received 28 January 2021; Accepted 1 June 2021; Published 14 July 2021

Academic Editor: Namhun Lee

Copyright ( $\odot 2021$ Bao-Chen Zhu et al. This is an open access article distributed under the Creative Commons Attribution License, which permits unrestricted use, distribution, and reproduction in any medium, provided the original work is properly cited.

Background. Aspirin is the first-line medication for prevention and treatment of coronary heart disease (CHD). However, long-term use of aspirin resulting in gastrointestinal mucosal injury and bleeding limits the regularity of medication. Xuesaitong is a marketed Chinese medicine contained main active component in Panax notoginseng saponins (PNS), which can significantly inhibit platelet aggregation in patients with CHD. Our previous studies have already showed that PNS could reduce the gastrointestinal mucosal injury caused by aspirin in preclinical study. However, there is a need for further clinical studies to evaluate synergy and attenuation effect of the combination. Methods. This trial is a prospectively planned, open-labeled, parallel-grouped, single-centered clinical trial. A total of eligible 480 participants will be randomly allocated into three groups: aspirin group, Xuesaitong group, and drug combination group at a ratio of $1: 1: 1$. The primary outcome is the change of platelet aggregation rate and calprotectin activity. Secondary outcomes include PAC-1, P-selectin, P2Y12, I-FABP activity, and fecal occult blood. Discussion. The results of the study are expected to provide evidence of high methodological and reporting quality on the synergy function of Xuesaitong and aspirin upon the antiplatelet and anti-gastrointestinal injury effect for CHD. It also provides an experimental basis for clinical rational drug combination therapy. Trial Registration. This trial was registered in the Chinese Clinical Trail Registry, ChiCTR2000036311, on 22 August 2020, http://www.chictr.org.cn/edit.aspx?pid=58798\&htm=4.

\section{Introduction}

Coronary heart disease (CHD) is the leading cause of death worldwide, with more than 1.5 million deaths each year $[1,2]$. Aspirin is a well-known baseline antiplatelet agent, serving as the first choice for the prevention and treatment of CHD. However, many studies have shown that the long-term administration of aspirin has the potential for significant side effects on the gastrointestinal tract causing mucosal lesions, bleeding, or peptic ulcers [3]. Although enteric-coated aspirin have been developed, 10\%-25\% long-term users still got peptic ulcers [4] and aspirin increases the risk of gastrointestinal bleeding by almost 3-fold [5]. These side effects limit the regularity of medication which increases the risk of cardiovascular accidents. Therefore, it is particularly important to prevent the occurrence of aspirin-induced gastrointestinal injury. While proton pump inhibitor (PPI) preparations are usually recommended to prevent aspirininduced gastrointestinal injury [4], especially for the elderly, the application of PPI damages the phospholipid layer in intestinal mucus, which may protect gastric mucosa but increase the risk of small intestinal injury [6]. Therefore, it is 
urgent to find a strategy to reduce aspirin-induced gastrointestinal injury.

Xuesaitong capsule is a marketed Chinese medicine with components of Panax notoginseng saponins (PNS), which is a mixture of ginsenosides $\mathrm{Rb} 1, \mathrm{Rg} 1, \mathrm{Rd}, \mathrm{Re}$, and notoginsenoside R1. It has been used in combination with aspirin in preventing cardiovascular disease for several years with a good clinical effect $[7,8]$. Our previous study has indicated that the combination could inhibit platelet activation caused by blood hypercoagulability and reduce platelet aggregation to prevent thrombosis $[9,10]$. These effects may rely on another finding from our team, which shows that aspirin and Xuesaitong could increase the area under the curve (AUC) and promote the absorption of each other [11, 12]. More interestingly, our research also found Xuesaitong attenuates expression of aspirin-induced tight junction protein and apoptosis of crypt epithelial cells in small intestine [13]. It means Xuesaitong may serve as a pharmacological treatment for aspirin-induced gastrointestinal injury. Since the dualdirectional regulation of Xuesaitong, it became the potential combination therapy to solve the clinical dilemma. All the preclinical evidences have elucidated the combination could enhance aspirin's efficacy and decrease aspirin-induced gastrointestinal injury. However, it still lacks high-level clinical evidence.

In this study, we conducted a randomized controlled noninferiority trial to explore whether the efficacy and safety of combination for $\mathrm{CHD}$ patients are superior to the single use.

\section{Methods}

2.1. Study Design and Setting. This is a prospectively planned, open-labeled, parallel-grouped, single-centered clinical trial. The trial will be conducted at the outpatient care of the Cardiology Department at the Dongzhimen Hospital, Beijing University of Chinese Medicine in China. The staff of the department will be informed of the trial and they will help researchers to recruit participants. The trial is conducted by the ethical principles laid down in the Declaration of Helsinki, the Guideline for Good Clinical Practice, and local laws. The informed consent form will be obtained from all participants before any trial-related procedures were conducted, enrolled after a complete and extensive description of the study. The trial was approved by the Ethics Committee of Dongzhimen Hospital, Beijing University of Chinese Medicine (Supplementary File 1). We present the protocol in accordance with the SPIRIT reporting checklist (Standard Protocol Items Recommendations for Interventional Trials) (Supplementary File 2).

In terms of research methods, we refer to and improve one of our previous experimental designs [14]. 480 participants will be randomly assigned to either an aspirin group, a Xuesaitong group, or drug combination group at a ratio of 1: 1: 1. Participants in the aspirin group will be administered aspirin $(100 \mathrm{mg}$, qd) orally $30 \mathrm{~min}$ after breakfast, participants in the Xuesaitong group will be administered Xuesaitong soft capsule (contains PNS and excipients; $200 \mathrm{mg}$, tid) orally $30 \mathrm{~min}$ after breakfast, and participants in the drug combination group will be administered both drugs above. The two drugs should be taken half an hour apart, and aspirin should be taken first. All treatment courses will last for 30 days. Blood samples will be acquired $30 \mathrm{~min}$ before administration on day 1 and 30 min after administration on day 30 . The volume of each blood sample is $4 \mathrm{~mL}$. And fecal samples will be collected on days 1 and 30. Details of patient follow-up are summarized in Table 1 and Figure 1.

2.2. Participants. The detailed inclusion, exclusion, and termination criteria are shown in Table 2.

2.3. Randomization. This is a single-center clinical trial with a sample size of 480 . They were randomly divided into three groups equally. The randomization code will be developed by the statistics office of Dongzhimen Hospital. The trial cases were separated into three arms. The research pharmacy will randomize the participants based on the master randomization list. A third party will join to ensure the allocation concealment.

2.4. Sample Size. The sample size was calculated based on the primary outcome (platelet aggregation rate). For power analysis, the primary outcome was assumed to be $38.72 \%$ in the aspirin group [15], and $37.79 \%$ in the Xuesaitong group [16]. Given a type I error at 0.05 and type II error at 0.2 , the required sample size was 131 per arm. Expecting $20 \%$ dropout rate, we planned to enroll 160 subjects.

2.5. Interventions. The dose and duration of medication in each group are illustrated in Table 3.

(1) Aspirin enteric-coated tablets (100 mg, qd) (Bayer S.p.A): a widely used antiplatelet medication

(2) Xuesaitong soft capsule (200 mg, tid) (Kunming Huarun Shenghuo Co. Ltd.): the main ingredient of the Xuesaitong soft capsule is Panax notoginseng saponins (PNS), which is commonly used in preventing and treating blood stasis, to prevent cardiovascular disease

\subsection{Combination}

(1) Participants are not allowed to take any kind of herbal or chemical medication which have the pharmacological effects of activating blood circulation, preventing platelet aggregation, or anti-inflammatory activity

(2) Participants with angina pectoris could take isosorbide mononitrate. In addition, participants could take other medications that have no known drugdrug interaction with aspirin or Xuesaitong soft capsule 
TABLE 1: Flowchart of a patient follow-up

\begin{tabular}{|c|c|c|c|c|}
\hline Group & Screening D-7 to D1 & D1 & D30 & D31 closeout \\
\hline Patient information & $\mathrm{X}$ & & & \\
\hline Physical examination & $\mathrm{X}$ & $\mathrm{X}$ & & $\mathrm{X}$ \\
\hline Vital signs & $\mathrm{X}$ & & & $\mathrm{X}$ \\
\hline Blood routine; serum biochemistry; urine routine; stool routine; coagulation routine & $\mathrm{X}^{1}$ & $\mathrm{X}^{1}$ & & $\mathrm{X}^{1}$ \\
\hline ECG & $\mathrm{X}$ & & & $\mathrm{X}$ \\
\hline Stool biomarkers (calprotectin; I-FABP) & & $\mathrm{X}$ & $\mathrm{X}$ & \\
\hline Blood biomarkers (platelet aggregation; P2Y12; PAC-1; P-selectin) & & $\mathrm{X}$ & $\mathrm{X}$ & \\
\hline
\end{tabular}

D: day; ECG: electrocardiogram; I-FABP: intestinal fatty acid-binding protein; PAC-1: platelet-associated complement $1 .{ }^{1}$ If the abnormality of this program is clinically significant, if it occurs before screening and at baseline, the subjects will be excluded directly. If it occurs during the observation period of administration, the retest will be followed up until the examination is normal or the abnormality is considered by the study doctor to be of no clinical significance.

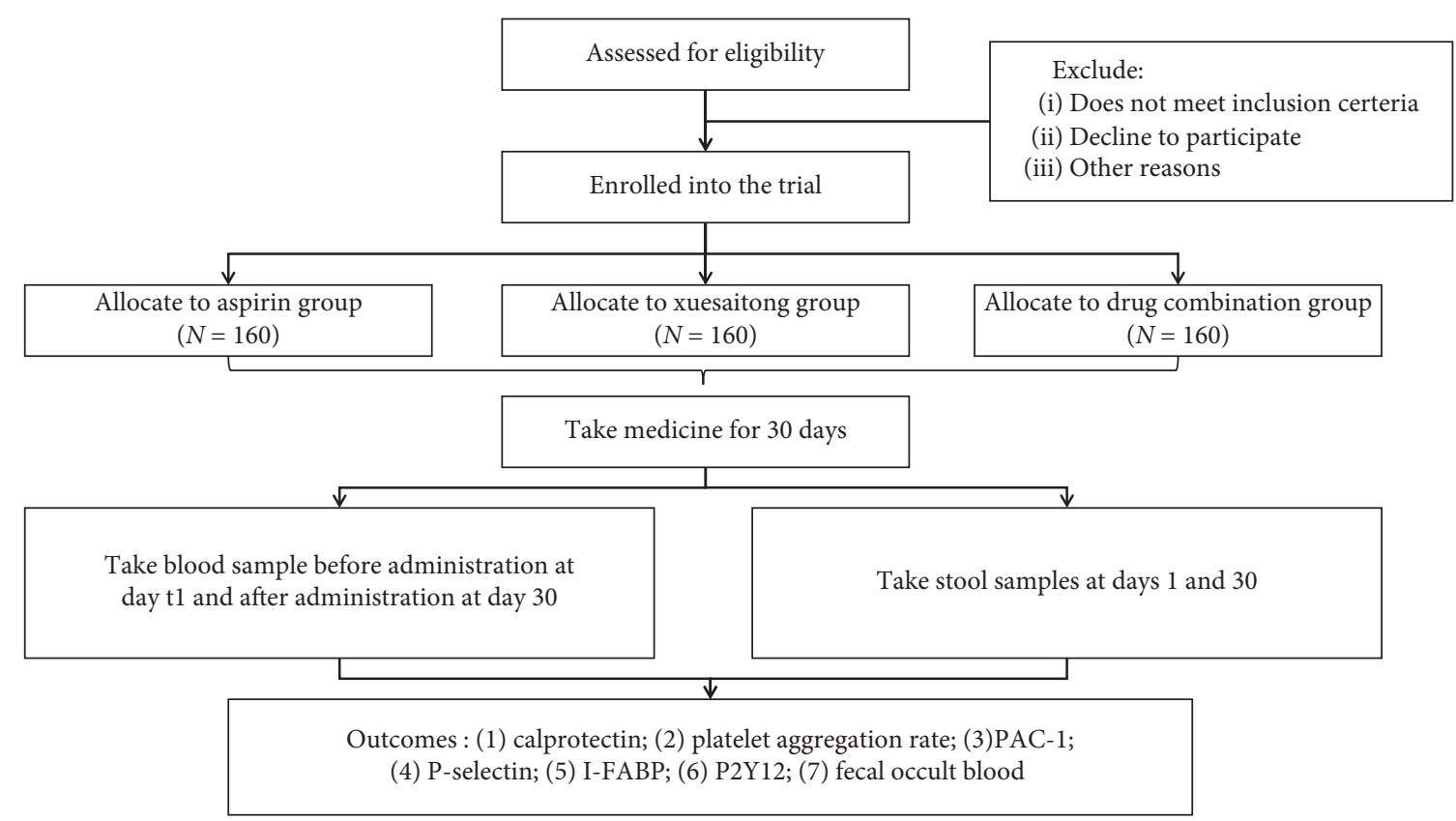

FIgURE 1: A schematic flowchart of the clinical trial.

\subsection{Sample Collection}

2.7.1. Aspirin Group. Participants should take aspirin with $200 \mathrm{~mL}$ of warm water on an empty stomach for 30 consecutive days.

2.7.2. Xuesaitong Group. Participants should take Xuesaitong soft capsule with $200 \mathrm{~mL}$ of warm water on an empty stomach for 30 consecutive days.

2.7.3. Drug Combination Group. Participants should take aspirin and Xuesaitong soft capsule on an empty stomach for 30 consecutive days. The interval between taking medicine is 30 minutes.

In all three groups, blood sampling will be carried out before administration on day 1 and after administration on day 30, and fecal samples will be collected on days 1 and 30.
2.8. Planned Outcomes and Measurements. The primary outcomes, secondary outcomes, and safety outcomes are summarized in Table 4.

2.9. Study Quality Control. Throughout the trial, each participant will be subject to safety monitoring. All the adverse events occurring will be transferred to the office of the clinical trial institution and Ethics Committee, who will review the events and adjudicate them with regards to causality.

We set DSMB (Data and Safety Monitoring Board) for this trial. The member of DSMB comprises physicians, clinical pharmacists, trial methodology experts, statistical experts, and members of the ethics committee, who will conduct risk assessment and safety analysis according to program termination criteria. Also, they will supervise the trial according to a manual of the procedure that has specific information and details for clinical research and laboratory 
TABLE 2: Inclusion, exclusion, and termination criteria.

Criteria

Inclusion criteria

(1) 45-75 years of age without gender restriction

(2) Meet the diagnostic criteria of CHD

(3) Meet the diagnostic criteria of grade I-II angina

(4) Meet the diagnostic criteria of blood stasis

(5) Meet the diagnostic criteria of stable angina pectoris

(6) Take regular aspirin (enteric-coated) for at least one year (the incidence of bleeding events, adverse events (AEs), and adverse reactions will be noted)

(7) Calprotectin in stool is above $50 \mu \mathrm{g} / \mathrm{g}$

(8) Before the study start, patients and their families are fully informed and voluntarily willing to sign an informed consent

\section{Exclusion criteria}

(1) Mental or physical disorders

(2) Pregnant, menstruating, and breastfeeding women

(3) Severe heart disease and severe cardiopulmonary dysfunction

(4) Severe primary diseases, such as cancer, liver and renal damage, and multiple organ failure

(5) Poorly controlled hypertension $(>160 / 100 \mathrm{mmHg}$ )

(6) Current participation in another clinical trial or participation in another clinical trial within three months

(7) Having underwent surgery within eight weeks

(8) Having drug allergy history or allergic constitution

(9) Diabetes

(10) Status epilepticus

(11) Unstable vital signs

(12) Combined with serious infection, infectious diseases

(13) Acute intestinal infection

(14) Unsuitability or poor compliance for this trial

Termination criteria

Participant termination

(1) Serious adverse events occur or having obvious abnormal value of the laboratory tests

(2) The investigators believe someone's trial needs to be terminated because of medical, safety, or GCP considerations

Trial termination

(1) Half of the participants have mild adverse events other than gastrointestinal reactions

(2) The investigators believe it is necessary to abort the trial for medical or safety purposes

TABle 3: Dose and duration of medication.

\begin{tabular}{lccc}
\hline Group & Medication & Dose and method & Duration \\
\hline $\begin{array}{l}\text { Aspirin group } \\
\text { Xuesaitong group }\end{array}$ & Enteric-coated aspirin & $100 \mathrm{mg}$, q.d., oral & 30 days \\
$\begin{array}{l}\text { Drug combination } \\
\text { group }\end{array}$ & Xuesaitong soft capsule & $200 \mathrm{mg}$, t.i.d., oral & 30 days \\
\hline
\end{tabular}

tasks. DSMB are fully blinded to the study intervention reviews and adjudicates adverse events.

2.10. Data Management and Statistical Analysis Plan. We use EDC system to collect the data. The system administrator establishes the eCRF and conducts logic verification and range checks for data values. Two investigators fulfill doubledata entry.

The analysis will be performed in a modified intentiontreat population, which included all randomized participants who received at least one dose of Xuesaitong or aspirin. Continuous variables are presented as the mean $\pm \mathrm{SD}$ and categorical variables as number and percentage. Comparisons between treatment groups were assessed using Student's $t$-test or Wilcoxon rank-sum test for continuous variables. Comparisons were assessed using the Fisher exact test for categorical variables. Data for patients who were lost to follow-up were censored at the time of the last contact. A multivariable Cox proportional-hazards regression analysis that included center and treatment as independent variables was conducted, and the results are expressed as hazard ratio (HR) with 95\% confidence interval (CI). The incidence of adverse events was compared using the chi-squared test. $P<0.05$ (two-sided) was considered to be statistically significant. All analyses were conducted using SPSS software 26.0 .

\section{Discussion}

To our knowledge, this is the first study to investigate the synergy effect of combination of Xuesaitong and aspirin. Aspirin is widely used in clinical practice for primary and secondary prevention of cardiovascular and thrombotic 
TABle 4: Clinical outcomes of the trial.

\begin{tabular}{l}
\hline Items \\
\hline Primary outcomes \\
Primary outcomes of improving efficacy \\
Platelet aggregation rate (platelet aggregation analyzer) \\
Primary outcomes of reducing gastrointestinal side effects \\
Change in calprotectin activity (ELISA) [17] \\
Secondary outcomes \\
Secondary outcomes of improving efficacy \\
(1) Change in PAC-1 activity (flow cytometry) \\
(2) Change in P-selectin activity (flow cytometry) \\
(3) Change in P2Y12 acceptor activity (flow cytometry) \\
Secondary outcomes of reducing gastrointestinal side effects \\
(1) Change in I-FABP activity (ELISA) [18] \\
(2) Change in fecal occult blood \\
Safety outcomes \\
(1) Serum biochemistry \\
(2) Blood routine \\
(3) Urine routine \\
(4) Stool routine \\
(5) ECG
\end{tabular}

cerebrovascular events $[1,2,19]$. However, the use of aspirin is well recognized to be associated with the risk of some gastrointestinal complications $[20,21]$. With the aging of society, the use of aspirin has continued to increase, and the gastrointestinal injury induced by aspirin, even at low doses, has become a clinical problem that needs attention. Some key strategies have been proposed to minimize the aspirininduced gastrointestinal injury, such as reducing the dose or frequency of aspirin administration, concomitant use of a gastroprotective drug, or even stopping administration of aspirin [22]. But these therapeutic regimens still remain in a dilemma between the prevention of thrombotic diseases and serious gastrointestinal complications. In order to solve this issue, it is urgent to find and establish therapeutic scheme that can not only effectively guarantee the clinical efficacy of aspirin but also prevent gastrointestinal injury. Since the dual-directional regulation of Xuesaitong, it became the potential combination of aspirin to solve the clinical dilemma above.

Xuesaitong, a marketed Chinese medicine with the main active component of PNS, has been widely and longterm used to prevent and treat hematological diseases and cardiovascular diseases [23]. Several clinic trials indicated that Xuesaitong could inhibit platelet activation in patients with blood hyperviscosity syndrome [24]. Interestingly, as for the widespread clinical application together with aspirin, many clinicians found its ability to protect against gastrointestinal injury. In view of this protective efficacy, some researches have shown that Xuesaitong could enhance the proliferative ability of human endothelial cells to heal the wound and stop bleeding [25]. Our previous studies demonstrated Xuesaitong can alleviate gastrointestinal injury caused by aspirin in rats [13]. Combined with HE staining results, our results indicate that the protective mechanism of Xuesaitong on aspirin-induced gastrointestinal injury may act through increasing VEGFA and protecting gastrointestinal mucosa. As for Xuesaitong's ability that both effectively guarantee the clinical efficacy of aspirin and prevent gastrointestinal injury, it is worthwhile to further study the mechanism of the combination of these two drugs and supply a rational combination. Since we have proved that aspirin and Xuesaitong could increase the AUC of each other [11, 12], this means the content of effective drugs in the blood increased. In order to achieve the same blood concentration, the intake of aspirin might be reduced, the adverse reaction might also reduce accordingly. To explore the possibility of reducing the dose of aspirin, it is worth making clear the efficacy and safety of the combination of aspirin and Xuesaitong. By using this randomized controlled noninferiority trial, determining the clinical efficacy indexes like aggregation rate, platelet membrane glycoprotein indexes, and gastrointestinal injury indexes like calcitonin activity and I-FABP allows us to find out whether the platelet aggregation in patients was excessively inhibited and whether the digestive tract injury caused by aspirin is alleviated under drug combination.

In conclusion, the results of this study are expected to provide evidence of high methodological quality on the synergy effect of combination of Xuesaitong and aspirin for CHD synergy effect of combination of Xuesaitong and aspirin.

$\begin{array}{ll}\text { Abbreviations } \\ \text { PNS: } & \text { Panax notoginseng saponins } \\ \text { CHD: } & \text { Coronary heart disease } \\ \text { PPI: } & \text { Proton pump inhibitor } \\ \text { AUC: } & \text { Area under the curve } \\ \text { I-FABP: } & \text { Intestinal fatty acid-binding protein } \\ \text { PAC-1: } & \text { Platelet-associated complement } 1 \\ \text { ECG: } & \text { Electrocardiogram } \\ \text { CCS: } & \text { Canadian Cardiovascular Society } \\ \text { DSMB: } & \text { Data and Safety Monitoring Board } \\ \text { HR: } & \text { Hazard ratio } \\ \text { CI: } & \text { Confidence interval } \\ \text { VEGFA: } & \text { Vascular endothelial growth factor A. }\end{array}$

\section{Data Availability}

The datasets used and analyzed in the current study are available from the corresponding author on reasonable request after the study completion.

\section{Additional Points}

Recruitment started on 2nd of May 2021 (Protocol version 1.0).

\section{Ethical Approval}

Ethical approval for this study was obtained through the Ethics Committee of Dongzhimen Hospital, Beijing University of Chinese Medicine (DZMEC-KY-2019-203). 


\section{Consent}

All participants will sign an informed consent. According to the privacy rules of clinical practice, investigators and physicians of the study will be the data confidentiality guarantors. The data used for analysis will be conducted anonymously under permission of participants. The results of this trial will be disseminated via publication.

\section{Disclosure}

Zhu Bao-chen and Xue Chun-miao are co-first authors. The funding body had no role in the study design or the decision to submit the manuscript for publication.

\section{Conflicts of Interest}

The authors declare that they have no conflicts of interest.

\section{Authors' Contributions}

Z. Wan-tong conceived the study and developed the first trial protocol. Z. Bao-chen designed the study and drafted the manuscript. X. Chun-miao and H. Guo-dong guided the research program. W. Xu-jie, L. Rui, and W. Wei-liang revised the article. L. Zhen-zhen, Z. Sha-sha, and Y. Wenhua edited the manuscript.

\section{Acknowledgments}

This study was supported by the fund of the National Natural Science Foundation of China (82004125) and Beijing University of Chinese Medicine (2020-JYB-XJSJJ-041).

\section{Supplementary Materials}

1: approval document of ethics. 2: SPIRIT reporting checklist (Standard Protocol Items Recommendations for Interventional Trials). (Supplementary Materials)

\section{References}

[1] Q. Hasanaj, B. J. Wilson, J. Little, Z. Montazeri, and J. C. Carroll, "Family history: impact on coronary heart disease risk assessment beyond guideline-defined factors," Public Health Genomics, vol. 16, no. 5, pp. 208-214, 2013.

[2] G. A. Roth, G. A. Roth, C. O Johnson et al., "The burden of cardiovascular diseases among US states, 1990-2016," JAMA Cardiology, vol. 3, no. 5, pp. 375-389, 2018.

[3] R. Guthrie, "Review and management of side effects associated with antiplatelet therapy for prevention of recurrent cerebrovascular events," Advances In Therapy, vol. 28, no. 6, pp. 473-482, 2011.

[4] W.-C. Chen, K.-H. Lin, Y.-T. Huang et al., "The risk of lower gastrointestinal bleeding in low-dose aspirin users," Alimentary Pharmacology \& Therapeutics, vol. 45, no. 12, pp. 1542-1550, 2017.

[5] K.-Y. Ji and F. L. Hu, "Interaction or relationship between Helicobacter pylori and non-steroidal anti-inflammatory drugs in upper gastrointestinal diseases," World Journal of Gastroenterology, vol. 12, no. 24, pp. 3789-3792, 2006.
[6] J. L. Wallace, "NSAID gastropathy and enteropathy: distinct pathogenesis likely necessitates distinct prevention strategies," British Journal of Pharmacology, vol. 165, no. 1, pp. 67-74, 2012.

[7] C. Z. Yang, Z. L. Li, and X. Y. Meng, "Therapeutic effects of aspirin and Xueshuantong patients with the drug resistance of ITGB3 gene polymorphism," Journal of Nanjing University of Traditional Chinese Medicine, vol. 5, pp. 493-496, 2017.

[8] S. Bent, "Herbal medicine in the United States: review of efficacy, safety and regulation," Journal of General Internal Medicine, vol. 23, no. 6, pp. 854-859, 2008.

[9] Z. Chen, T. Lu, X. Yue et al., "Neuroprotective effect of ginsenoside $\mathrm{Rb} 1$ on glutamate-induced neurotoxicity: with emphasis on autophagy," Neuroscience Letters, vol. 482, no. 3 , pp. 264-268, 2010.

[10] T. Kuhlmann, L. Remington, B. Maruschak et al., "Nogo-A is a reliable oligodendroglial marker in adult human and mouse CNS and in demyelinated lesions," Journal of Neuropathology \& Experimental Neurology, vol. 66, no. 3, pp. 238-246, 2007.

[11] Z. Tian, H. Pang, S. Du et al., "Effect of Panax notoginseng saponins on the pharmacokinetics of aspirin in rats," Journal of Chromatography B, vol. 1040, pp. 136-143, 2017.

[12] Z. Tian, H. Pang, Q. Zhang et al., "Effect of aspirin on the pharmacokinetics and absorption of panax notoginseng saponins," Journal of Chromatography B, vol. 1075, pp. 25-33, 2018.

[13] B. Zhu, W. Zhang, Y. Lu et al., "Network pharmacology-based identification of protective mechanism of Panax Notoginseng Saponins on Aspirin induced gastrointestinal injury," Biomedicine \& Pharmacotherapy, vol. 105, pp. 159-166, 2018.

[14] W. Zhang, B. Zhu, W. Cao, R. Li, S. Wang, and R. Gao, "Research on the mechanism of drug-drug interaction between salvianolate injection and aspirin based on the metabolic enzyme and PK-PD model: study protocol for a PK-PD trial," Trials, vol. 19, no. 1, p. 491, 2018.

[15] H. Li, Y. P. Sun, and X. H. Tan, "Effects of different doses of clopidogrel combined with aspirin on hemorheology, platelet aggregation rate and safety in patients with unstable angina pectoris (In Chinese)," Contemporary Medicine, vol. 1, pp. 176-178, 2020.

[16] Y. Liu and K. Zhang, "Effects of sanqi tongshu capsule on serum levels of TXB2 and IL- 6 and platlet activity of aspirin resistance patients with ischemic stroke (in Chinese)," Journal of Liaoning University of Traditional Chinese Medicine, vol. 11, pp. 21-22, 2013.

[17] G. O. Altug, K. Tuncay, M. Balahan et al., "Role of fecal calprotectin in the assessment of intestinal inflammation in children with familial Mediterranean fever," International Journal of Rheumatic Diseases, vol. 21, pp. 1844-1848, 2018.

[18] M. Voth, T. Lustenberger, B. Relja, and I. Marzi, "Is I-FABP not only a marker for the detection abdominal injury but also of hemorrhagic shock in severely injured trauma patients?" World Journal of Emergency Surgery, vol. 14, no. 1, p. 49, 2019.

[19] P. B. Gorelick and S. M. Weisman, "Risk of hemorrhagic stroke with aspirin use," Stroke, vol. 36, no. 8, pp. 1801-1807, 2005.

[20] G. L. Moneta, “Aspirin in the primary and secondary prevention of vascular disease: collaborative meta-analysis of individual participant data from randomised trials," Lancet, vol. 373, pp. 1849-1860, 2009.

[21] J. Backman, L. Yerges-Armstrong, R. Horenstein et al., "Prospective evaluation of genetic variation in platelet endothelial aggregation receptor 1 reveals aspirin-dependent 
effects on platelet aggregation pathways," Clinical And Translational Science, vol. 10, no. 2, pp. 102-109, 2017.

[22] M. Sugimoto, M. Nishino, C. Kodaira et al., "Impact of acid inhibition on esophageal mucosal injury induced by low-dose aspirin," Digestion, vol. 85, no. 1, pp. 9-17, 2012.

[23] B. R. Yang, S. C. Yuen, G. Y. Fan, W.-H. Cong, S.-W. Leung, and S. M.-Y. Lee, "Identification of certain Panax species to be potential substitutes for Panax notoginseng in hemostatic treatments," Pharmacological Research, vol. 134, pp. 1-15, 2018.

[24] S. G. Wei, L. Q. Meng, and R. Y. Huang, "Effect of Panax notoginseng saponins on serum neuronal specific enolase and rehabilitation in patients with cerebral hemorrhage," Phytotherapy Research, vol. 27, pp. 159-162, 2007.

[25] D. Wang, Q. Jie, B. Liu et al., "Saponin extract from Panax notoginseng promotesangiogenesis through AMPK- and eNOS-dependent pathways in HUVECs," Molecular Medicine Reports, vol. 16, no. 4, pp. 5211-5218, 2017. 